\title{
New Approaches For Augmented UHF RFID Textile Yarn
}

\author{
Sofia Benouakta \\ Université de Lyon, Université Claude \\ Bernard Lyon 1, CNRS \\ Laboratoire Ampère \\ Villeurbanne, France \\ sofia.benouakta@univ-lyon1.fr
}

\author{
Florin Hutu \\ Université de Lyon, \\ INSA Lyon, Inria, CITI \\ Villeurbanne, France \\ florin-doru.hutu@insa-lyon.fr
}

\author{
Yvan Duroc \\ Université de Lyon, Université Claude \\ Bernard Lyon 1, CNRS \\ Laboratoire Ampère \\ Villeurbanne, France \\ yvan.duroc@univ-lyon1.fr
}

\begin{abstract}
In the context of smart textiles, this paper addresses the evolution of the UHF RFID technology towards novel functionalities such as physical parameters sensing and tag's integration with the identified object since the fabrication step. More precisely, the first part is dedicated to the presentation of physical parameters sensing such as temperature and humidity starting UHF RFID yarns. The second part of this paper deals with the design and the characterization of a helical antenna able to support elongation efforts and so offering a better integration with the textile wire.
\end{abstract}

Keywords-RFID textile yarn, helical antenna, temperature sensor, humidity sensor

\section{INTRODUCTION}

The popular concept of Internet of Things (IoT) is most commonly achieved using wireless systems. Radio Frequency IDentification (RFID) is one solution among the plethora of radiofrequency low power consumption protocols designed for the IoT. Already deployed in wide and various range of other applications [1-2], the ultrahigh-frequency (UHF) RFID systems are of particular interest also in this context. For RFID communication, the concept of wearables has been growing in the last years. The major requirement when designing wearable antennas is to make the technology invisible to the users, passing from the traditional idea of a tag associated to an object toward the concept of a tag integrated into the object itself [3]. Thus, in complement to the current approaches to manufacture 3D and wearable RFID tags [4-7], and based on the industrial E-thread technology [8], the approach presented in [9] re-invented the RFID tag form factor. The E-thread technology proposes a slender and conformable RFID tag able to be wound in a spool.

In this work, the ambition is to propose approaches for adding new capabilities such as wireless sensing functionalities [10] and a robustness in terms of elasticity.

The paper is organized as following. Section II describes a concept enabling to add a sensing function to the RFID yarn. Two examples are illustrating the concept for temperature sensing and humidity sensing respectively. Section III proposes the use of a helical antenna in order to achieve an elastic UHF RFID yarn. Finally, section IV presents conclusions and perspectives of this work.

\section{UHF RFID TEXTILE YARN WITH SENSING FUNCTIONS}

Based on the E-Thread technology, an UHF RFID textile yarn was designed in [9]. The RFID yarn is made of a dipole antenna connected to an RFID chip and embedded in a textile yarn. The antenna is composed of a driven element of length $L_{1}=170 \mathrm{~mm}$, tap by a shorted stub of length $L_{2}=33 \mathrm{~mm}$ in a point off center. The stub and the driven element have both diameter of $D=120 \mu \mathrm{m}$ and are separated by a distance $s=440 \mu \mathrm{m}$ from center to center (Fig. 1). The resulted structure presents $14 \mathrm{~m}$ of read range from $800 \mathrm{MHz}$ to $920 \mathrm{MHz}$, a realized gain of $2 \mathrm{dBi}$ and can be easily used as primary raw in fabrics.

The UHF RFID textile yarn achieves good performance when used for identification. The proposed idea is to transform the current solution in order to add sensing capabilities.

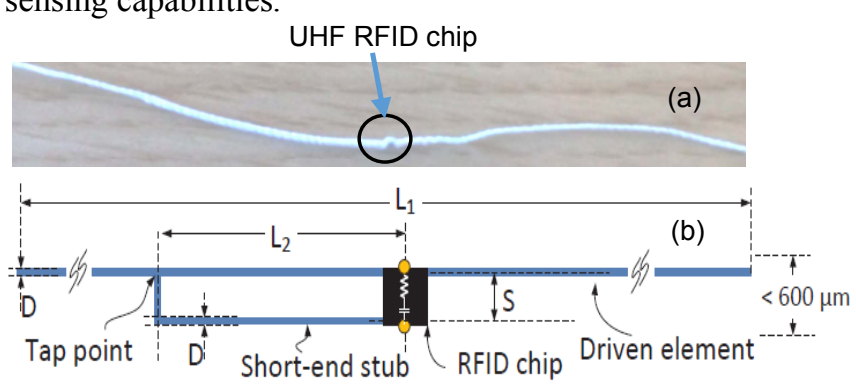

Fig. 1. (a) Manufactured RFID UHF textile yarn; (b) Topology of the RFID UHF tag

\section{A. RFID UHF Textile Yarn Sensing Methodology}

In order to manufacture an RFID sensor yarn, the UHF RFID yarn described previously is associated to a component or a material that is sensitive to the ambient environment physical properties such as temperature or humidity. Any variation in the physical quantity will lead to a change in the sensor's tag performance, notably due to the impedance matching variation. The minimum transmitted power to activate the tag will vary and enables to deduce the corresponding variation of the captured information. For this purpose, an UHF RFID tag yarn without sensor is used as a reference (Fig. 2). 


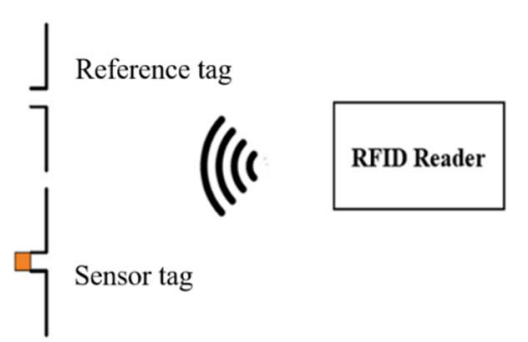

Fig. 2. Measurement method using an RFID reader, a classical RFID reference dipole yarn and RFID sensor yarn

Using the extended Friis equation in $\mathrm{dBm}$, the transmitted power to the sensor yarn $P_{t}$ can be expressed by (1) and the transmitted power to the reference tag yarn $P_{0}$ is expressed by (2):

$$
\begin{aligned}
& P_{t}=P_{t a g}-G_{t}-L_{L O S}-\chi-G_{t a g} \tau \\
& P_{0}=P_{t a g}-G_{t}-L_{L O S}-\chi-G_{t a g} \tau_{0}
\end{aligned}
$$

where:

$P_{\text {tag }}(\mathrm{dBm})$ is the tag sensitivity, i.e., the activation power of the tag;

$G_{t}(d B i)$ is the reader's antenna gain, taking in consideration the impedance matching;

$L_{L O S}$ is the propagation path loss;

$\chi$ is the polarization matching coefficient;

$G_{\text {tag }}(d B i)$ is the tag antenna's gain;

$\tau$ is the power transmission coefficient for the sensor tag; $\tau_{0}$ is the power transmission coefficient for reference tag. The differential transmitted power can be written as:

$$
\Delta P=P_{t}-P_{0}=G_{t a g}\left(\tau-\tau_{0}\right)
$$

with

$$
\tau=\frac{4 R_{\text {chip }} R_{\text {ant }}}{\left|Z_{\text {chip }}+Z_{\text {ant }}\right|^{2}} \quad \text { and } \quad \tau_{0}=\frac{4 R_{\text {chip }} R_{0}}{\left|Z_{\text {chip }}+Z_{0}\right|^{2}}
$$

where:

$Z_{\text {chip }}=R_{\text {chip }}+j X_{\text {chip }}$ is the impedance of the chip;

$Z_{0}=R_{0}+j X_{0}$ is the impedance of the reference antenna;

$Z_{\text {ant }}=R_{\text {ant }}+j X_{\text {ant }}$ is the impedance of the sensor tag antenna.

Without loss of generality, the following data are considered in the two next sections for the performance illustration: $G_{t}=5.29 \mathrm{dBi}, P_{\text {tag }}=-17 \mathrm{dBm}$ and $G_{\mathrm{tag}}=$ $2 \mathrm{dBi}$. Moreover, the channel is assumed as a free space channel and the distance between reader and tags is fixed at 1 meter. Otherwise, all the simulations are performed using CST Microwave Studio ${ }^{\circledR} 2018$.

Finally, it is worth noting that the proposed method is very relevant because the measure is not sensitive to the polarization and the propagation channel. Indeed, the reference tag and the sensor tag can be considered in first approximation submitted to the same (or at least very similar) polarization and same propagation channel (even if more complex than simple free space attenuation).

\section{B. RFID Temperature Sensing Yarn}

In order to design the RFID temperature sensor yarn, a thermistor is connected in series with the RFID chip of the RFID yarn. The thermistor resistance is decreasing when the temperature is increasing. The structure is shown in Fig. 3. Here, the dimensions are optimized in order to achieve the impedance matching: $L_{1}=170 \mathrm{~mm}, L_{2}=$ $33 \mathrm{~mm}, D=120 \mu \mathrm{m}$ and $S=440 \mu \mathrm{m}$. Commercial thermistor NTC [11] was considered where the resistance value is increased from $1.54 \Omega$ to $56 \Omega$ in order to simulate the temperature variation.

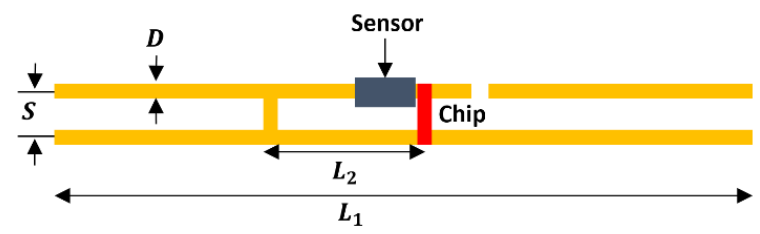

Fig. 3. Topology of the RFID temperature sensor yarn

From simulation results, Fig. 4 shows that the obtained realized gain of the RFID sensor yarn is increased with temperature due to the change in the impedance matching.

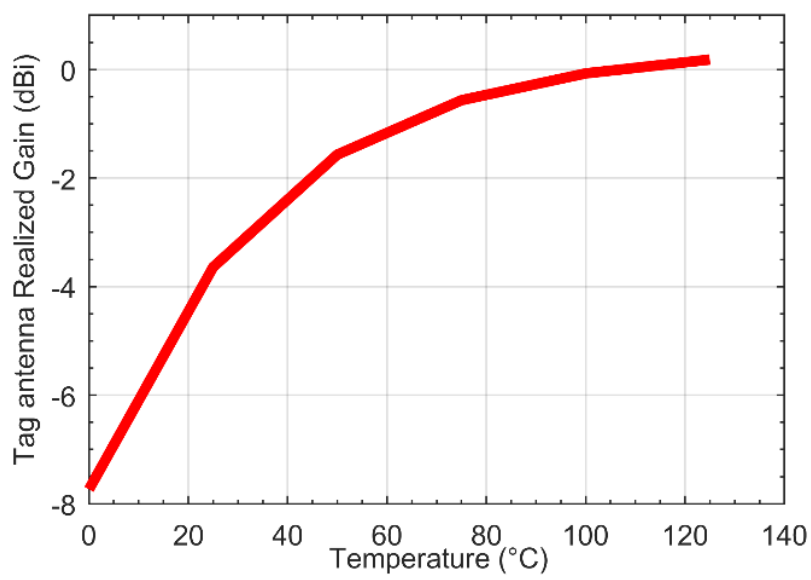

Fig. 4. Realized gain of the RFID temperature sensor yarn from different values of temperature

Fig. 5 shows that an increase in the temperature leads to a decrease in the differential power between the reference tag yarn and the temperature sensor yarn.

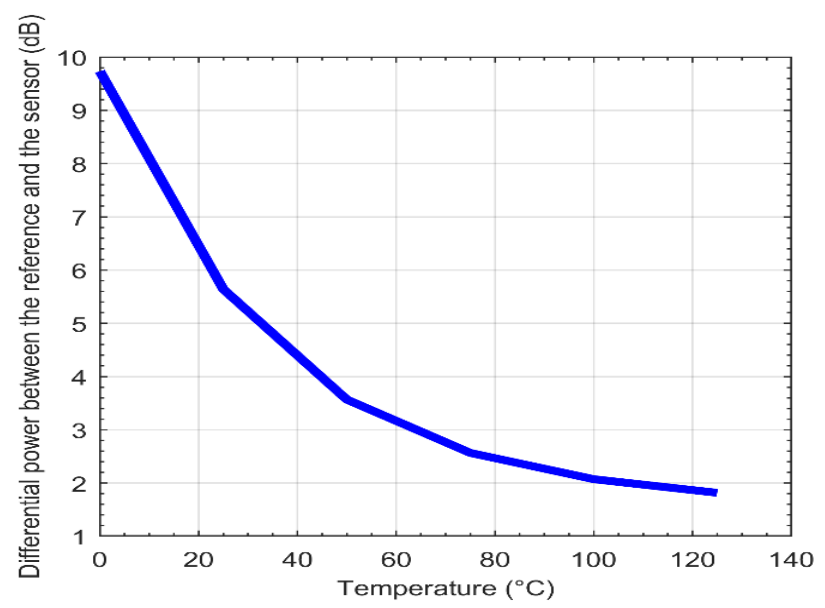

Fig. 5. Differential power between the reference RFID tag yarn and the temperature sensor yarn transmitted by the RFID reader 


\section{RFID Humidity Sensing Yarn}

The proposed relative humidity sensor relies on the same topology as shown by Fig. 6. Inspired from [12], the humidity is sensed by inserting a capacitor $\mathrm{C}$ between the two wires exploiting the gamma match section. For this design, the considered dimensions are the following: $L_{1}=$ $136 \mathrm{~mm}, L_{2}=21 \mathrm{~mm}, L_{3}=10.5 \mathrm{~mm}, \mathrm{~s}=440 \mu \mathrm{m}$ and $a=a^{\prime}=60 \mu \mathrm{m}$. Note that the dimensions were optimized in order to achieve the impedance matching [13].

In order to simulate a change in humidity, a capacitor was shunted with a chip according to a commercial humidity sensor as in [14].

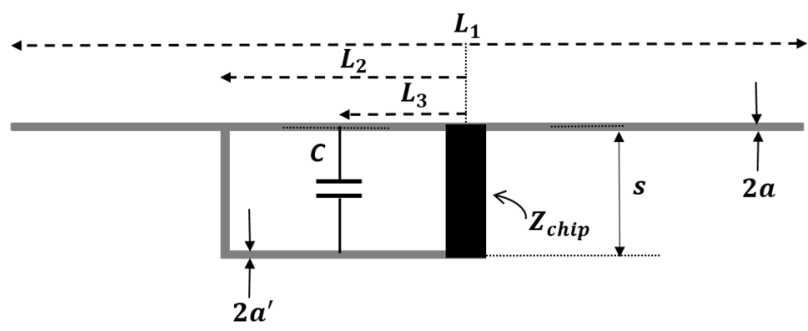

Fig. 6. Topology of the capacitive based humidity sensor.

Fig. 7. shows the transmission coefficient for different capacitance values. The resonance frequency is shifted to low frequencies in the UHF RFID band, without affecting the realized gain.

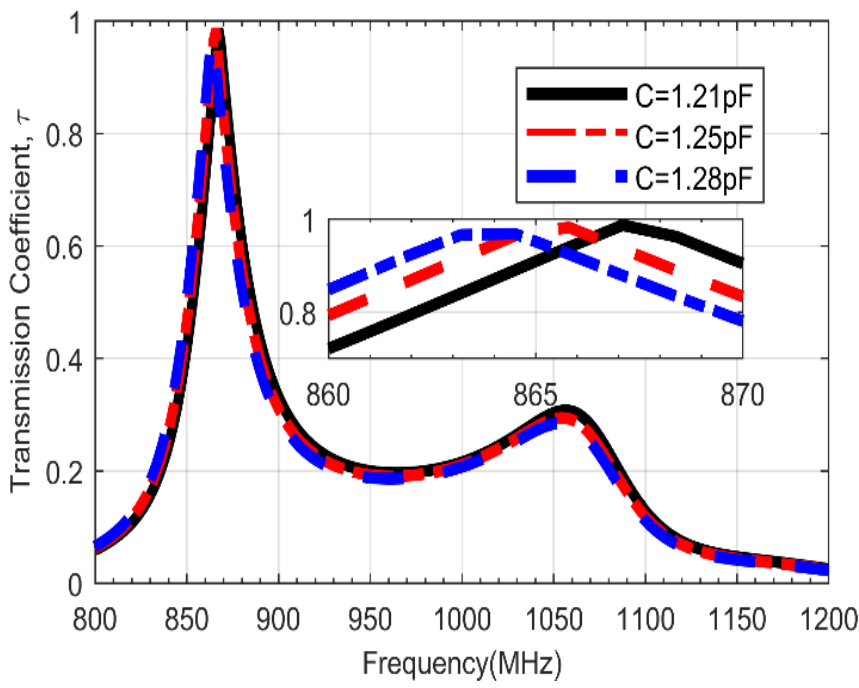

Fig. 7. Transmission coefficient of the capacitive based humidity sensor for different capacitive values

Consequently, with the same method than in the case of the temperature sensor, the differential transmitted power can be calculated for each capacitance value at $867 \mathrm{MHz}$. It is worth noting that another approach in this case could be to take into consideration the frequency shift for achieving the measurement.

\section{ELASTIC UHF RFID YARN}

\section{A. Helical Dipole Antenna}

The performance of the yarn depends strongly on the robustness of the antenna characteristics to the operating scenario mechanical solicitations. In order to overcome this constraint, a helical dipole antenna geometry is proposed. The helical antenna is composed of winded conductor constituting the radiating element. The performance of helical antenna is defined by its geometrical parameters: the diameter $D$, the spacing between the turns $s$, the number of turns $N$, the half-length $h$, the conductor radius $a$ (Fig. 8).

\section{B. Helical Antenna Design considerations}

The helical dipole antenna has to operate in the European UHF frequency band $(865.5-867.5 \mathrm{MHz})$. The RFID chip associated to the helical antenna is assumed to have a typical input impedance value of 15 - j $150 \Omega$; the value corresponds to the impedance of the Monza M4 RFID chip [15].

Regarding the dimensions of the helical antenna, in order to be embedded in a slender yarn, the diameter of the helical dipole $D$ and the conductor radius $a$ are fixed: $D=$ $1 \mathrm{~mm}$ and $a=0.1 \mathrm{~mm}$. Consequently, the spacing between turns, the axial half-length and the number of turns are the geometrical parameters that have to be optimized in order to achieve the UHF resonance and the impedance matching with the RFID chip.

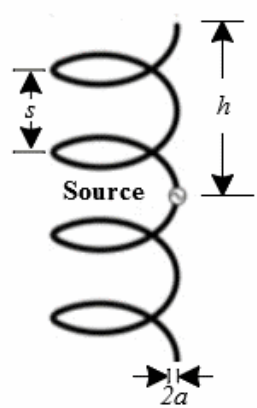

(a)

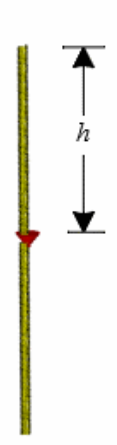

(b)

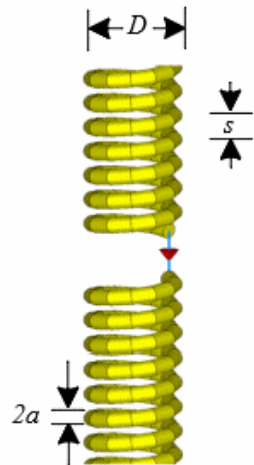

(c)
Fig. 8. Helical dipole antenna: (a) illustration of the geometrical parameters; (b) full view of the antenna in CST; (c) zoomed view around the feeding point.

\section{Helical Antenna Impedance Matching and Optimization}

Starting from initial parameters: $N=60 ; h=18 \mathrm{~mm}$, preliminary simulations showed that the spacing between two adjacent turns $s$ has a strong impact on the antenna input impedance whereas the number of turns $N$ and the antenna's half-length $h$ act more on the resonance frequency. Thus, the optimization is performed by starting to achieve the impedance matching before varying $N$ and $h$ to achieve the operating frequency (while maintaining the impedance matching) [16].

Finally, considering all the assumptions and constraints described before, the optimal parameters of the helical dipole antenna have been found such as: $s=0.3 \mathrm{~mm}, N=$ 80 , and $h=24 \mathrm{~mm}$.

Fig. 9 shows the reflection coefficient of the helical dipole antenna. The impedance matching with the RFID chip is achieved at the resonance frequency of $867 \mathrm{MHz}$. The bandwidth (defined at $-10 \mathrm{~dB}$ ) is from $863.54 \mathrm{MHz}$ up to $870.47 \mathrm{MHz}$ and equal to $6.94 \mathrm{MHz}$. 


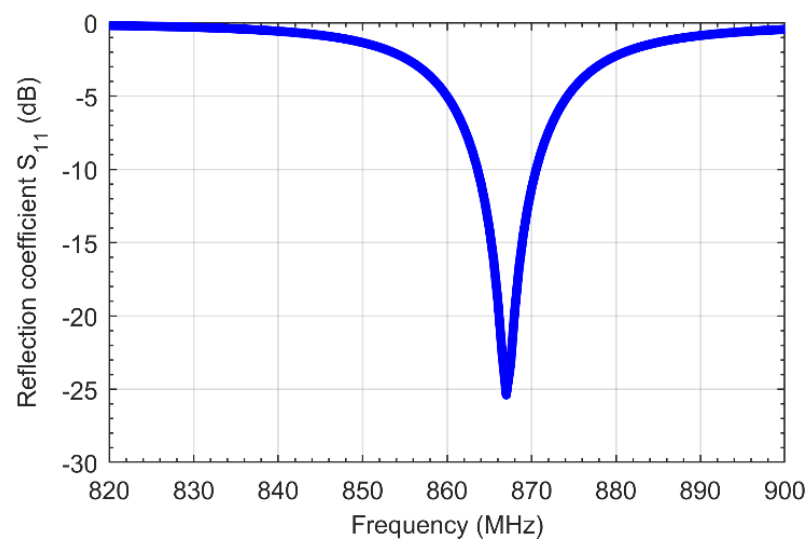

Fig. 9. Reflection coefficient of the helical dipole antenna after optimization

\section{Robustness of the Helical Antenna to Axial Elongation:}

In order to evaluate the robustness of the helical dipole antenna considering an axial elongation, the half-length $h$ is extended symmetrically for the two sides, from the initial value $h=h_{0}=24 \mathrm{~mm}$ up to $30 \mathrm{~mm}$. In this case, the number of turns is obviously kept constant. Table 1 summarizes the considered lengths and highlights the corresponding relative stretching defined as:

$$
E[\%]=\frac{h-h_{0}}{h_{0}} \times 100 \%
$$

Fig.10 shows the impact on the reflection coefficient when $h$ increases. The resonance frequency is shifted toward lower frequencies. Furthermore, the absolute value of the reflection coefficient decreases with an enlargement of the bandwidth. For an axial stretching until 16\%, the frequency bandwidth (defined at $-10 \mathrm{~dB}$ ) is large enough for ensuring the RFID communication link in UHF European band.

\begin{tabular}{|c|c|c|c|c|}
\hline h $[\mathrm{mm}]$ & 24 & 26 & 28 & 30 \\
\hline E $[\%]$ & 0 & 8.33 & 16 & 25 \\
\hline
\end{tabular}

TABLE I. RELATIVE ELONGATION FOR EACH CONSIDERED LENGTH

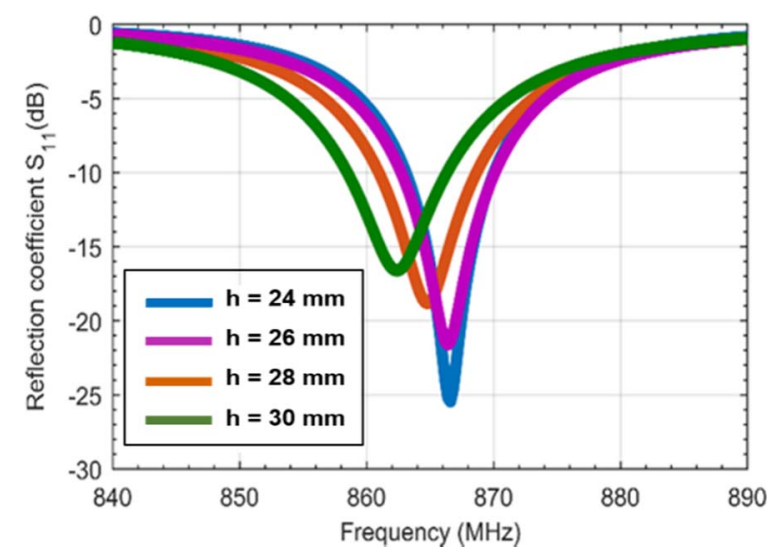

Fig. 10. Reflection coefficient of the helical dipole antenna for different lengths from $18 \mathrm{~mm}$ to $24 \mathrm{~mm}$ for achieving resonance frequency in the RFID UHF band.

The helical dipole antenna is a good alternative to achieve an elastic RFID UHF yarn. In addition, the helical antenna has also potential for achieving sensing functions through a dielectric material introduced as a core.

\section{E. Helical antenna prototype}

A first prototype of helical antenna for UHF RFID yarn was fabricated according to Primo1D company's assembling method. The prototype is presented in Fig. 11. During the assembling process, a wrapping material is added around the coils to achieve insulation and avoid short circuits. However, this step was not considered during simulation. Consequently, the antenna's performance and implicitly the read range are degraded. Using the commercial test bench [17], the reading distance at $868 \mathrm{MHz}$ is about 1 meter.

This first prototype validates the use of helical antenna in the context of RFID UHF tags. Moreover, it hightlights further design considerations and technology constraints such as dimensions imposed by manufacturing process, the wrapping material and the dieletric core.

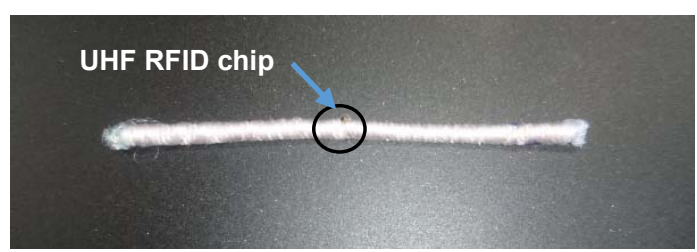

Fig.11. Prototype of the UHF RFID tag based on helical antenna

\section{CONCLUSION}

In this paper, new capabilities have been added to the UHF RFID textile yarn based on the E-Thread technology. A method has been proposed to implement sensing capabilities into the UHF RFID textile yarn. The method is based on the estimated differential transmitted power between a RFID reference yarn and the RFID sensor yarn. The proposed concept is applied on two types of sensors: temperature and humidity. In addition, in order to obtain an elastic RFID UHF textile yarn, a helical antenna was proposed. The results showed robustness to elongation up to $16 \%$ from the original length. A first prototype of a RFID UHF tag based on helical antenna was manufactured.

\section{ACKNOWLEDGMENT}

This work has been supported by « La Région Auvergne-Rhône-Alpes » France and was performed in collaboration with Primo1D company.

\section{REFERENCES}

[1] L. Yan, Y. Zhang, L. Yang, and H. Ning, "The intemet of things: from RFID to the next-generation pervasive networked systems," Wireless Networks and Mobile Communications. CRC Press, 2008.

[2] Y. Duroc and S. Tedjini, "RFID: a key technology for humanity," Comptes Rendus Physique, vol. 19, pp. 64-71, January-February 2018.

[3] L. Corchia, G. Monti and L. Tarricone, "Wearable antennas: nontextile versus fully textile solutions," IEEE Antennas and Propagation Magazine, vol. 61, no. 2, pp. 71-83, April 2019.

[4] K. Koski, L. Sydänheimo, Y. Rahmat-Samii, and L. Ukkonen, "Fundamental characteristics of electro-textiles in wearable UHF RFID patch antennas for body-centric sensing systems," IEEE 
Transactions on Antennas and Propagation, vol. 62, no. 12, pp. 6454-6462, December 2014.

[5] D. Patron, W. Mongan, T.P. Kurtzweg, A. Fontecchio, G. Dion, E.K. Anday, and K.R. Dandekar, "On the use of knitted antennas and inductively coupled RFID tags for wearable applications," IEEE Transactions on Biomedical Circuits and Systems, vol. 10, pp. 1047-1057, December 2016.

[6] Q. Liu, T. Le, S. He, and M.M. Tentzeris, "Button-shaped radiofrequency identification tag combining three-dimensional and inkjet printing technologies," IET Microwave, Antennas, Propagation, vol. 10, pp. 737-741, May 2016.

[7] X. Chen, S. Ma, L. Ukkonen, T. Björninen, and J. Virkki, "Antennas and antenna-electronics interfaces made of conductive yarn and paint for cost-effective wearable RFIDs and sensors," in Proc. IEEE MTT-S International Microwave Symposium, Honololu, HI, USA, June 4-9, 2017, pp. 827-830.

[8] J. Brun, "Method for assembling a microelectronic chip element on a wire element, and installation enabling assembly to be performed," US Patent App. 14/375,859, January, 2015.

[9] G. Andía, "Slenderly and conformable passive UHF RFID yarn," in Proc. IEEE International Conference on RFID, Phoenix, AZ, USA, May 9-11, 2017, pp. 130-136.

[10] S. Lemey, S. Agneessens, and H. Rogier, "Wearable smart objects," IEEE Microwave Magazine, pp. 83-100, Sept.-Oct. 2018.

[11] Murata products- product details https://www.murata.com/products/productdetail?partno=NCP15XC 220E03RC

[12] S. Koley, H. Pant and L. Murmu, "Half-elliptical UWB planar monopole with reconfigurable slot antenna for cognitive radio frontend," Journal of Circuits, Systems, and Computers, vol. 27, no. 13,pp. 1-13, 2018.

[13] S. Koley, S. Benouakta, F. Hutu, and Y. Duroc, "Passive UHF RFID yarn for yelative humidity sensing application," in Proc. European Conference on Antennas and Propagation, Krakow, Poland, 31 March - 5 April, 2019.

[14] C. Zhao, M. Qin and Q. Huang, "A fully packaged CMOS interdigital capacitive humidity sensor with polysilicon heaters," IEEE Sensors Journal, vol. 11, no. 11, pp. 2986-2992, November 2011.

[15] Impinj. Impinj UHF RFID product downloads - monza tag chips. [Online]. Available: http://www.impinj.com/products/tag-chips/

[16] S. Benouakta, S. Koley, F. Hutu, Y. Duroc, "Conception d'une antenne hélice pour fil textile RFID UHF extensible,". Journées Nationales Micro-ondes (JNM 2019), Caen, France, May 14-17, 2019.

[17] Voyantic. Tagformance pro. [Online]. Available: https://voyantic.com/products/tagformance-pro 